REVISTA MATEMÁTTCA de la

Universidad Complutense de Madrid

Volumen 6, número I; 1993.

http://dx.doi.org/10.5209/rev_REMA.1993.v6.n1.17843

\title{
Dunford-Pettis-like Properties of Continuous Vector Function Spaces
}

\author{
Jesus M.F. Castillo and Fernando Sanchez
}

\begin{abstract}
In this paper, the structure of some operator ideals $\mathscr{A}$ defined on continuous function spaces is studied. Conditions are considered under which " $T \in \mathcal{A}$ " and "the representing measure of $T$ takes values in $\mathscr{A}^{\prime \prime}$ are equivalent for the scales of p-converging $\left(C_{p}\right)$ and weaklyp-compact $\left(W_{p}\right)$ operators. The scale $C_{p}$ is intermediate between the ideals $C_{t}=u$ (unconditionally summing operators), and $C_{\alpha}=\mathcal{B}$ (completely continuous operators), which have been studied by several authors (Bombal, Cembranos, Rodriguez-Salinas, Saab). The dual scale $W_{p}$ is intermediate between the ideals $\chi$ (compact operators) and $W_{\mathrm{oo}}=W$ (weakly compact operators), and the results presented have a close connection with those of Diestel, Núñez and Seifert.
\end{abstract}

\section{PRELIMINARIES}

In this paper, $\mathrm{B}(\Sigma, X)$ denotes the space of all bounded $X$-valued $\Sigma$ measurable functions; if $1 \leq p \leq \infty, p^{*}$ denotes the conjugate number of $p$; if $p=1, l_{p^{*}}$ plays the role of $c_{0}$.

1.1. Definition. A sequence $\left(x_{n}\right)$ in a Banach space $X$ is said to be weakly-p-summable $(I \leq p \leq \infty)$ if $\left(x^{*} x_{n}\right) \in l_{p}$ for all $x^{*} \in X^{*}$, or equivalently, if there is a constant $C>0$ such that

1991 Mathematics Subject Classification: 46E15, 46B28, 46B25.

Editorial Complutense. Madrid, 1993. 


$$
\sup _{n}\left\|\sum_{k=1}^{n} \xi_{k} x_{k}\right\| \leq C \cdot\left\|\left(\xi_{n}\right)\right\|_{p_{p}}
$$

for any sequence $\left(\xi_{n}\right) \in l_{p^{*}}$. We shall denote by $w_{p}\left(\left(x_{n}\right)_{n}\right)$ the infimum of those constants $C$.

We shall say that $\left(x_{n}\right)$ is weakly-p-convergent to $x \in X$ if $\left(x_{n}-x\right)$ is

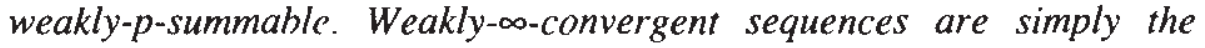
weakly convergent sequences.

1.2. Definition. Let $1 \leq p \leq \infty$. An operator $T \in \mathscr{L}(X, Y)$ is said to be $p$ convergent if it transforms weakly-p-summable sequences into norm null sequences. We shall denote by $C_{p}$ the class of $p$-convergent operators.

When $p=\infty$ this definition gives the ideal $B$ of completely continuous operators, that is to say, those transforming weakly null sequences into norm null sequences. When $p=1$, it is easy to verify that $C_{1}=U$, the ideal of unconditionally summing operators, i.e., those transforming weakly-1summable sequences into summable ones. Obviously $C_{q} \subset C_{p}$ when $p<q$.

The scale of $C_{p}$ ideals are intermediate between the ideals $B$ and $U$. It is clear (from the definition) that $C_{p}$ are injective operator ideals, and, since any separable Banach space is a quotient of $l_{1}$, they are not surjective. On the other hand, it is easy to see that $C_{p}$ is closed: let $\left(T_{n}\right)$ be a sequence of $p$-converging operators with limit (in the operator norm) $T$. If $\left(x_{n}\right)$ is a weakly $p$-summable sequence and $\varepsilon>0$, then $\left\|T x_{n}\right\| \leq \varepsilon\left\|x_{n}\right\|+$ $\| T_{k} x_{n} \mid \leq 2 \varepsilon$ and $\left(T x_{n}\right)$ is norm null.

1.3. Definition. $A$ bounded set $K$ in a Banach space is said to be relatively weakly-p-compact $(1 \leq p \leq \infty)$ if every sequence in $K$ has a weakly-p-convergent sub-sequence. An operator $T \in \mathscr{L}(X, Y)$ is said to be weakly-p-compact, $1 \leq p \leq \infty$, if $T\left(B_{X}\right)$ is relatively weakly-p-compact. We shall denote by $W_{p}$ the ideal of weakly-p-compact operators.

The $W_{p}$ operators are meant to be a gradations of the class of weakly 
compact operators. It is clear that $W_{\infty}=W$ (weakly compact operators), and it is easy to see that $i d(X) \in W_{1}$ if and only if $X$ is finite dimensional. Obviously $W_{p} \subset W_{q}$ when $p<q$.

The ideals $W_{p}$ are injective and surjective but not closed. The ideal $W_{1}$ is not closed since $W_{1} \neq W_{1}^{2}=K$, the ideal of compact operator (see [14]). To see $W_{p}$ is not closed for $p>1$, we apply [14, Prop. 1.6] to the diagram:

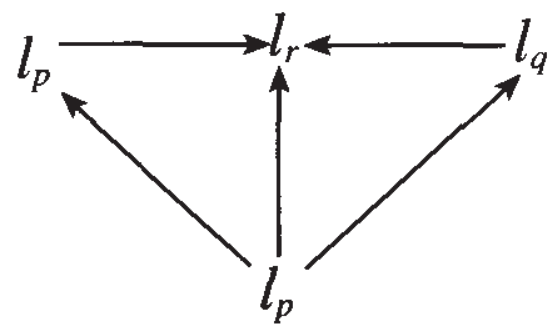

for $1<p<r<q$. The left arrow is the identity and the right arrow is the inclusion, which belongs to $W_{q^{*}}$. If this operator ideal was closed, the middle inclusion should also be in $W_{q^{*}}$, which is not, since $C_{p} \circ W_{p}=K$ and

\subsection{Proposition. Let $1<p<\infty$, then id $\left(l_{p}\right) \in W_{p^{*}}$.}

Proof. Let $\left(x_{n}\right)$ be a bounded sequence in $l_{p}$. It admits a weakly convergent sub-sequence $\left(x_{k}\right)$. Let $x$ be its weak limit, and let us call $y_{k}=x_{k}-x$. If $\left(y_{k}\right)$ is norm null, we have finished. If not, and we have $\left\|y_{k}\right\| \geq \varepsilon>0$ for some sub-sequence, applying the Bessaga-Pelczynski selection principle, we obtain a new sub-sequence, equivalent to the canonical basis $\left(e_{n}\right)$ of $l_{p}$, which is weakly $p^{*}$-summable.

An easy consequence is:

1.5. Proposition. $\mathscr{L}\left(l_{p^{*}}, X\right)=K\left(l_{p^{*}}, X\right)$ if and only if $i d(X) \in C_{p}$.

Moreover, an operator $T$ belongs to $C_{p}(X, Y)$ if and only if for each $j \in \mathscr{P}\left(l_{p}, X\right)$ the composition $T \circ j$ is compact. From this and the proof of (2.5) we obtain

1.6. Proposition. If $T \in W_{p}(X, Y)$ then $T^{*} \in C_{r}\left(Y^{*}, X^{*}\right)$ for all $r<p^{*}$. 
1.7. Corollary. Let $1<p<\infty$, id $\left(l_{p}\right) \in C_{r}$ for all $r<p^{*}$.

\section{Remarks.}

1. The progression expressed by (1.7) suddenly breaks down when $p<1$, due to [17], where it is shown that a weakly-1-summable sequence $\left(x_{n}\right)$ exists in each $l_{p}, p<1$, for which $\left\|x_{n}\right\|_{p} \rightarrow+\infty$.

2. Regarding Proposition 1.5, this result is equivalently to Pitt's lemma: $\mathscr{L}\left(l_{p}, l_{q}\right)=K\left(l_{p}, l_{q}\right)$ if and only if $p>q$.

For $L_{p}$ spaces the situation is:

\subsection{Proposition.}

a) If $2 \leq p<\infty$ then id $\left(L_{p}\right) \in W_{2}$.

b) If $I<p<2$ then $i d\left(L_{p}\right) \in W_{p^{*}}$.

Proof. Part a) can be obtained by using the Kadec-Pelczynski alternative: every normalized weakly null sequence in $L_{p}$ has a subsequence equivalent either to the unit vector basis of $l_{p}$ or the unit vector basis of $l_{2}$.

Part b) follows from a standard duality argument. If $\left(x_{n}\right)$ is a normalized weakly null sequence in $L_{p}$ and $\left(x_{k}\right)$ is a basic sub-sequence of $\left(x_{n}\right)$, consider a bounded sequence $\left(y_{k}\right)$ of biorthogonal functionals in $L_{p^{*}}$ and (again) the Kadec-Pelczynski alternative.

1.9. Examples. (See [21] for details). We shall abbreviate $i d(X) \in C_{p}$ (resp. $i d(X) \in W_{p}$ ) by saying $X \in C_{p}$ (resp. $X \in W_{p}$ ). (1.7)).

a) If $1 \leq p<\infty, l_{p} \in C_{r}$ for $1 \leq r<p^{*}$, and $l_{p} \in W_{p^{*}}$ for $1<p<\infty$ (see (1.4) and 
b) If $1 \leq p<\infty, L_{p}(\mu) \in C_{r}$ for $r<\min \left(2, p^{*}\right)$. If $1<p<\infty, L_{p}(\mu) \in W_{r}$ for $r=\max \left(2, p^{*}\right)$ (see $(1.8)$ and $(1.6)$ ).

c) Tsirelson's space $T$ is such that $T \in C_{p}$ for all $p \neq \infty$ (see [7]).

d) Tsirelson's dual space $T^{*}$ is such that $T^{*} \in W_{p}$ for all $p>1$ (see [7]).

e) Super-reflexive spaces belong to some class $W_{p}$ and, consequently, to some class $C_{q}$ (see [6]).

f) If $X, l_{r} \in W_{p}$ then so does $l_{r}(X)$ (see [8]).

It is well-known [12] that every operator $T$ from $C(K, X)$ to $Y$ has a finitely additive representing measure $m$ of bounded semi-variation, defined on the Borel $\sigma$-field $\Sigma$ of $K$ and with values in $\mathscr{L}\left(X, Y^{* *}\right)$, in such a way that

$$
T(f)=\int f d m, \quad(f \in C(K, X))
$$

If $m: B o(K) \rightarrow \mathscr{L}(X, Y)$ is a finitely additive measure, we shall denote by $|m|$ its semi-variation. One says that $|m|$ is continuous at $\varnothing$ if it has a control mesasure: a countably additive positive measure $\lambda$ on $B o(K)$ such that

$$
\lim _{\lambda(A) \rightarrow 0}|m|(A)=0
$$

1.10. Proposition. When $T \in W(C(K, X), Y)$, its associated representing measure $m$ is countably additive and verifies the following two conditions:

a) $|m|$ is continuous at $\varnothing$, and

b) for each $A \in B o(K), m(A) \in W(X, Y)$.

Thus, it seems natural to ask which properties pass from $T$ to $m$ and viceversa. 


\section{OPERATORS AND MEASURES}

By mimicry of the proofs made in [3], [4] and [20] for the cases $p=1, \infty$ one can easily obtain:

2.1. Proposition. Let $T \in C_{\rho}(C(K, X), Y)$, and let $m$ its representing measure. Then:

a) $|m|$ is continuous at $\varnothing$, and

b) for each $A \in B o(K), m(A) \in C_{p}(X, Y)$.

Nevertheless, these two conditions a) and b) do not characterize $C_{p}$ operators. In [1], there is shown an operator $T$ from $C\left([0,1], c_{0}\right)$ to $c_{0}$ which is not in $C_{1}$ but is such that its representing measure $m$ has continuous semi-variation at $\varnothing$, and $m(A)$ is a compact operator for any Borel set $A \subset[0,1]$.

2.2. Proposition. Let $T \in \mathscr{L}(C(K, X), Y)$ have a representing measure $m$ satisfying: and

a) $|m|$ is continuous at $\varnothing$ and admits a discrete control measure,

b) for each $A \in B o(K), m(A) \in C_{p}(X, Y)$.

Then $T \in C_{p}(X, Y)$.

Since every Radon measure over a dispersed compact set is discrete (see $[16, \S 2])$, it follows that:

2.3. Corollary. If $K$ is dispersed and $T \in \mathscr{L}(C(K, X), Y)$ is such that its representing measure $m$ satisfies:

a) $|m|$ is continuous at $\varnothing$, and

b) for each $A \in B o(K), m(A) \in C_{p}(X, Y)$, then $T \in C_{p}(X, Y)$. 
Corollary (2.3) asserts that (2.1) is an equivalence when $K$ is dispersed. We can also expect an equivalence when some condition is imposed on $X$.

2.4. Proposition. Let $l \leq p \leq \infty$. The following are equivalent:

a) $i d(X) \in C_{p}$.

b) Given any compact space $K$ and any Banach space $Y$, an operator $T \in C_{p}(C(K, X), Y)$ if and only if its representing measure satisfies

i) $|m|$ is continuous at $\varnothing$, and

ii) for each $A \in B o(K), m(A) \in C_{p}$.

Concerning the dual scale of weakly-p-compact operators, we have:

2.5. Lemma. Let $T \in \mathscr{H}(C(K, X), Y)$ and $p \geq 1$. The following are equivalent $\left(\hat{T}\right.$ is the restriction to $\mathrm{B}(\Sigma, X)$ of the operator $\left.T^{* *}\right)$ :
a) $T \in W_{p}(C(K, X), Y)$,
b) $\hat{T} \in W_{p}(\mathrm{~B}(\Sigma, X), Y)$,
c) $T^{* *} \in W_{p}\left(C(K, X)^{* *}, Y\right)$.

Proof. Since $T \in W(A, B)$ if and only if $T^{*}$ (or any of its iterated duals) is weak*-to-weak continuous, and the unit ball of $A$ is weak*-dense in the unit ball of $A^{* *}$, we have:

$$
T^{* *}\left(\boldsymbol{B}_{A} \ldots\right)=T^{* *}\left(\boldsymbol{B}_{A}^{-\left(A{ }^{*} A^{*}\right)}\right) \subset T\left(\dot{\left.B_{A}\right)}\right.
$$

from which the result follows.

That immediately gives:

2.6. Proposition. Let $T \in W_{p}(C(K, X), Y), p \geq 1$. Its associated measure verifies: 
a) $|m|$ is continuous at $\varnothing$, and

b) for each $A \in B o(K), m(A) \in W_{p}(X, Y)$.

The converse is not true; see the comments after (2.1).

\section{DUNFORD-PETTIS-LIKE PROPERTIES}

A Banach space $X$ is said to have the Dunford-Pettis property if any weakly compact operator $T: X \rightarrow Y$ transforms weakly compact sets of $X$ into norm compact sets of $Y$. This property can be described by means of the inclusion $W(X, Y) \subset \mathrm{B}(X, Y)=C_{\infty}(X, Y)$. We can weaken this requirement in the following manner:

3.1. Definition. Let $l \leq p \leq \infty$. We shall say that a Banach space $X$ has the Dunford-Pettis property of order $p$ (in short $D P P_{p}$ ) if the inclusion $W(X, Y) \subset C_{p}(X, Y)$ holds for any Banach space $Y$.

Obviously $D P P_{p}$ implies $D P P_{q}$ when $q<p$. Also, $D P P=D P P_{\infty}$ and every Banach space has $D P P_{1}$. It follows from the definition that if $i d(X) \in C_{p}$ then $X$ has $D P P_{p}$, and that if $i d(X) \in W_{p}$ then $X$ does not have $D P P_{p}$. The following result contains analytical and geometrical characterizations of the $D P P_{p}$.

3.2. Proposition. For a given Banach space $X$, the following are equivalent:

a) $X$ has $D P P_{p}(1 \leq p \leq \infty)$.

b) If $\left(x_{n}\right)$ is a weakly-p-summable sequence of $X$ and $\left(x_{n}^{*}\right)$ is weakly null in $X^{*}$ then $\left(x_{n}^{*} x_{n}\right) \rightarrow 0$.

c) Every weakly compact operator $T: X \rightarrow Y$ transforms weakly-pcompact sets of $X$ into norm compact sets of $Y$. 
Proof. The proof of the equivalence between (a) and (b) is obtained as in [21]. We prove the equivalence of (a) and (c).

(c) $\Rightarrow$ (a): Consider $T: X \rightarrow Y$ a weakly compact operator, and $\left(x_{i n}\right)$ a weakly-p-summable sequence in $X$. We form the set:

$$
\operatorname{conv}_{p},\left\{\left(x_{n}\right)\right\}=\left\{\sum_{n=1}^{\infty} \lambda_{n} x_{n}: \Sigma_{n} \mid \lambda_{n} p^{p} \leq 1\right\}
$$

which we shall refer to as the $p^{*}$-convex hull of $\left(x_{n}\right)$. Clearly, conv $v_{p^{*}}\left(x_{n}\right)$, the continuous image by the natural operator associated to $\left(x_{n}\right)$ of the unit ball of $l_{p^{*}}$, is a weakly-p-compact set. Since $T \in C_{p}$ and $l_{p} \in W_{p^{*}}$, $T\left(\operatorname{conv}_{p^{*}}\left(x_{n}\right)\right)$ is compact, and $\left(T x_{n}\right)$ is norm-null.

(a) $\Rightarrow$ (c): If $A$ is a weakly-p-compact set of $X$, then for each bounded sequence $\left(z_{m}\right)$ of $A$ there is a point $z \in A$, and a sub-sequence $\left(z_{n}\right)$, such that $\left(z_{n}-z\right)$ is weakly-p-summable. We set $\left(x_{n}\right)=\left(z_{n}-z\right)$, and apply to this sequence the preceding argument, to conclude that $\left(T x_{n}\right)$ admits a norm null sub-sequence.

3.3. Examples, The following examples are immediate after (1.9). In fact, these results give the optimum values of $p$.

a) $C(K)$ and $L_{1}$ have the $D P P$, and therefore the $D P P_{p}$ for all $p$.

b) If $1<r<\infty, l_{r}$ has the $D P P_{p}$ for $p<r^{*}$.

c) If $1<r<\infty, L_{r}(\mu)$ has the $D P P_{p}$ for $p<\min \left(2, r^{*}\right)$.

d) Tsirelson's space $T$ has $D P P_{p}$ for all $p<\infty$. However, since $T$ is reflexive, it does not have $D P P$.

e) Tsirelson's dual space $T^{*}$ does not have $D P P_{p}$ for any $p>1$.

Coming back to continuous vector function spaces, we have: 

$D P P_{p}$.

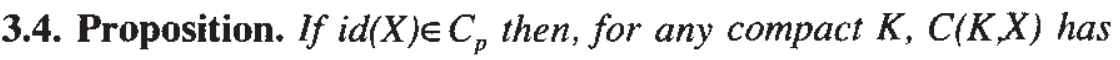

Proof. Let $T \in W(C(K, X), Y)$. If $\left(f_{n}\right)$ is a weakly-p-summable sequence in $C(K, X)$, then for each $t \in K$, the sequence $\left(f_{n}(t)\right)$ is also weakly-psummable in $X$, and thus it is norm null. The sequence $\left(T f_{n}\right)$ is also null by $[5$, Th. 2.1$]$.

3.5. Corollary. Given any compact space $K$ and $1<p<\infty, C\left(K, l_{p}\right)$ has $D P P_{r}$ for all $r<p^{*}$; it does not have $D P P_{p^{*}}$.

A "limit case" is provided by Tsirelson's spaces (compare this result with (3.13)):

3.6. Corollary. If $T$ denotes Tsirelson's space then, given any compact space $K$ and $I<p<\infty, C\left(K, T^{*}\right)$ has $D P P_{p}$ but not $D P P$.

Now, we see what happens if we replace the condition " $i d(X) \in C_{p}$ " by the weaker "X has the DPP ".

3.7. Example. Talagrand's construction of a Banach space $X$ having $D P P$ but such that $C(K, X)$ does not have $D P P$ (see [22]), can be modified in such a form that we obtain Banach spaces $T_{p}(p>1)$ having $D P P$, and such that $C\left(K, T_{p}\right)$ does not have $D P P_{p}$. Talagrand's original example corresponds to $T_{2}$.

What can be said about $C(K, X)$ when $X$ simply has $D P P_{p}$ ? The following theory was developed in [4] and [2] for DPP.

3.8. Definition. An operator $T: C(K, X) \rightarrow Y$, whose associated measure $m$ has continuous semi-variation at $\varnothing$, is said to be almost- $C_{p}$ if, for each weakly-p-summable sequence $\left(x_{n}\right)$ of $X$ and each bounded sequence $\left(\phi_{n}\right)$ of $C(K)$, the sequence $T\left(\phi_{n} x_{n}\right)$ converges to 0 in $Y$. Obviously, $C_{p}$-operators are almost- $C_{p}$. 
3.9. Theorem. The following are equivalent:

a) $X$ has $D P P_{p}$

b) For each compact space $K$, every weakly compact operator $T: C(K, X) \rightarrow Y$ is almost $-C_{p}$.

c) Every weakly compact operator $T: C([0,1], X) \rightarrow Y$ is almost $-C_{p}$.

d) Every weakly compact operator $T: C([0,1], X) \rightarrow c_{0}$ is almost- $C_{p}$.

(The proof is exactly as [2, Th. 5]).

3.10. Corollary ([10, [13]). Let $1 \leq p \leq \infty$. For a dispersed compact space $K$, the following are equivalent:

a) $C(K, X)$ has $D P P_{p}$.

b) $X$ has $D P P_{p}$

Proof. Implication $a) \Rightarrow b$ ) follows from (3.9). Conversely, if $T \in W(C(K, X), Y)$ with representing measure $m$, for each Borel set $A \subset K$, $m(A) \in W(X, Y) \subset C_{p}(X, Y)$, since $X$ has $D P P_{p^{*}}$. Applying (2.3), we obtain $T \in C_{p}$.

Concerning the scales $W_{p}$, Diestel and Seifert proved in [11] that weakly compact operators defined on $C(K)$ spaces are Banach-Saks operators. Recall that an operator $T \in \mathscr{L}(X, Y)$ is said to be Banach-Saks (in short $T \in B S$ ) if any bounded sequence $\left(x_{n}\right)$ of $X$ admits a sub-sequence $\left(x_{m}\right)$ such that $\left(T x_{m}\right)$ has norm-convergent arithmetic means.

Núñez [18] extended this result to $C(K, X)$ spaces showing that, when $X$ is super-reflexive, then weakly compact operators defined on $C(K, X)$ are Banach-Saks. In [9], it is shown a vector measure whose range is not a weakly-p-compact set for any $p$. That example provides a weakly compact operator $T$, defined on a certain $C(K)$ space, which, for every $p$, does not belong to $W_{p}$, showing that, in general, $X \in W_{p}$ does not imply 
$W(C(K, X), Y) \subset W_{p}(C(K, X), Y)$, and therefore, that in some sense, the result of Diestel and Seifert cannot be improved.

Despite that negative result, when $K$ is a dispersed compact space, some positive results can be obtained:

3.11. Proposition. If $X \in W_{p}$ then $W\left(c_{0}(X), Y\right) \subset W_{p}\left(c_{0}(X), Y\right)$.

Proof. Let $T \in W\left(c_{0}(X), Y\right)$ and let $\left(f_{n}\right)$ be a bounded sequence in $c_{0}(X)$. Let $\varepsilon>0$. For each $n \in \mathbb{N}$, a number $p_{n}$ exists so that $\left\|f_{n}(k)\right\| \leq \varepsilon 2^{-n}$ for $k \geq p_{n^{*}}$

We write $f_{n}=f_{n}^{d}+f_{n}^{i}$, where

$$
f_{n}^{i}=\left(f_{n}(1), \ldots, f_{n}\left(p_{n}-1\right), 0,0, \ldots\right)
$$

and

$$
\left.f_{n}^{d}=\left(0,0, \ldots, 0, f_{n}\left(p_{n}\right), f_{n}+1\right), \ldots\right)
$$

Since $\left\|f_{n}^{d}\right\| \rightarrow 0$, it is enough to see that $\left.T\left(f_{n}^{i}\right)\right)$ admits a weakly-pconvergent sub-sequence. For each $k \in \mathbb{N}$, there exists $q_{k}$ such that $w_{p}\left(\left(f_{n}^{i}(k)-x_{k}\right)_{n \geq q_{k}}\right) \leq \lambda$ (the constant $\lambda$ can be chosen uniformly [15]).

We determine inductively a sequence of indices $\left(q_{s(n)}\right)$ as follows:

$$
q_{s(0)}=q_{1} \text { and } q_{s(n+1)} \geq \max \left\{q_{k}: k \leq p\left(q_{s(n)}\right)\right\}
$$

so that $p\left(q_{s(n+1)}\right)>p\left(q_{s(n)}\right)$, and consider the sub-sequence $f_{n}^{i}=f_{q_{s a t}}^{i}$.

We now write $f_{n}^{i}=s_{n}+t_{n}$ where

$$
t_{n}=\left(0,0,0, \ldots f_{n}^{i}\left(p_{q_{n}}\right), \ldots . f_{n}^{i}\left(p_{q_{n+1}}\right), 0,0, \ldots\right),
$$

so that it is the continuous image of a block basic sequence constructed against the canonical basis of $c_{0}$. We see that, passing to a sub-sequence if necessary, $\left(T t_{n}\right)$ converges to 0 . 
The sequence

$$
\left(z_{n}\right)= \begin{cases}z_{n}(k)=f_{n}^{i}(k) & \text { if } k \leq p\left(q_{s(n-1)}\right), \\ z_{n}(k)=0 & \text { otherwise }\end{cases}
$$

however, is the continuous image of (a part of) the summing basis $\left(e_{1}+\ldots+e_{n}\right)_{n}$ of $c_{0}$.

If we set $x=\left(x_{1}, x_{2}, x_{3}, \ldots\right) \in l_{\infty}(X)$, we see, passing again to a subsequence if necessary, that $\left\|T z_{n}-T^{* *} x\right\| \leq 2^{-n}$.

Finally, if $\left(\xi_{n}\right)$ is a finite sequence in the unit ball of $l_{p^{*}}$ then

$$
\begin{aligned}
\left\|\Sigma_{n} \xi_{n}\left(T s_{n}-T^{* *} x\right)\right\| & \leq\left\|\Sigma_{n} \xi_{n}\left(T s_{n}-T z_{n}+T z_{n}-T^{* *} x\right)\right\| \\
& \leq\|T\| \cdot\left\|\Sigma_{n} \xi_{n}\left(s_{n}-z_{n}\right)\right\|+1 \leq \lambda \cdot\|T\|+1,
\end{aligned}
$$

thus finishing the proof.

Remark. If the choice of indices indicated in the proof is not possible because the sequence $\left(p_{n}\right)$ does not go to infinity, then we would be working in a finite product space $X^{n}$; if it is because the sequence of $q_{n}$ stops at $q$, then we shall follow the same reasoning as in the last part with the sub-sequence, $f_{q}, f_{q+1}, \ldots$

3.12. Theorem. Let $K$ be a dispersed compact space and $X \in W_{p}$. Then:

$$
W(C(K, X), Y) \subset W_{p}(C(K, X), Y)
$$

Proof. Let $T \in W(C(K, X), Y)$ and let $\left(f_{n}\right)$ be a bounded sequence in $C(K, X)$. By a standard argument we can assume $K$ to be countable, $K=\left\{t_{1}, t_{2}, \ldots\right\}$. Since $m$ (the associated measure of $T$ ) has continuous semi- 
variation at $\varnothing$, a $p_{n}$ exists for each $n \in \mathbb{N}$ such that, if we set $\mathrm{B}_{k}=\left\{t_{j}: j \geq k\right\}$, then $|m|\left(\mathbf{B}_{p, n}\right) \leq 2^{n}$.

Once more we write $f_{n}=f_{n}^{d}+f_{n}^{i}$ where $f_{n}^{d}$ converges to 0 and $f_{n}^{i}$ is eventually zero. Since $f_{n}^{i}$ is a bounded sequence in a space isomorphic to some $c_{0}(\mathbb{N}, X)$, the proof of (3.11) applies.

3.13. Corollary. If $K$ is a dispersed compact space and $T^{*}$ denotes Tsirelson's dual space, then $W\left(C\left(K, T^{*}\right), Y\right) \subset W_{p}\left(C\left(K, T^{*}\right), Y\right)$ for all $p>1$.

A sufficient condition on $X$ which guarantees the inclusion $W(C(K, X), Y) \subset W_{p}(C(K, X), Y)$ is given by:

3.14. Theorem. If $X$ does not contain $c_{0}$ finitely represented, then

$$
W(C(K), X) \subset W_{2}(C(K), X)
$$

Proof. If $X$ does not contain $c_{0}$ finitely represented, then there is a $p>1$ such that $\mathscr{L}(C(K), X)=W(C(K), X) \subset \Pi_{p}(C(K), X)$ by [19]. But each $p$ summing operator sub-factorizes through an $L_{p}$-space, which gives $\Pi_{p} \subset W_{2}$ when $p \geq 2$, and thus for all $p$.

The hypothesis is not necessary: just consider Tsirelson's space $T^{*}$.

\section{FINAL REMARKS AND FURTHER QUESTIONS}

Results (3.12) and (3.14) suggest the following problems:

Problem $K$. Characterize the compacts $K$ such that for any Banach space $X$

$$
W(C(K), X) \subset W_{2}(C(K), X) .
$$

Problem $X$. Characterize those Banach spaces $X$ such that for any compact $K$ 


$$
W(C(K), X) \subset W_{2}(C(K), X)
$$

Notice that the hypothesis of (3.14) is not necessary: if $K$ is dispersed, then $W\left(C\left(K, T^{*}\right), Y\right) \subset W_{p}\left(C\left(K, T^{*}\right), Y\right)$ for all $p>1$ and $T^{*}$ is not, for any $p<\infty$, of cotype $p$.

An application could be the following conjecture, essentially due to Drewnowski: Is it true that $\mathscr{L}\left(l_{2}, X\right)=K\left(l_{2}, X\right) \Leftrightarrow \mathscr{L}\left(l_{\infty}, X\right)=K\left(l_{\infty}, X\right)$ ? One implication is clear. To see the other, notice that $X \in C_{2}$ and $\mathscr{L}\left(l_{2}, X\right)=$ $K\left(l_{2}, X\right)$ are equivalent. Since $C_{2} \circ W_{2}=K$, and since $X \in C_{2}$ implies $\mathscr{L}\left(l_{\infty}, X\right)=$ $W\left(l_{\infty}, X\right)$, the question is whether a) Banach spaces $X \in C_{2}$ satisfy affirmatively Problem $X$, or b) the Stone-Cech compactification of $\mathbb{N}, \beta \mathbb{N}$, satisfies affirmatively Problem K.

Another unsolved question about the relationships between $T$ and $m$ is the following: Is it true that if $K$ is a dispersed compact, and, for every Borel set $A$, the operator $m(A) \in W_{p}$, then $T \in W_{p}$ ?

The example in [9] mentioned before (3.11) shows that the hypothesis " $K$ dispersed" cannot be removed.

Besides this, Núnez proved in [18] that if $T: C(K, X) \rightarrow Y, K$ is dispersed and, for every Borel set $A$, the operator $m(A) \in B S$, then $T \in B S$. The connection with Núnez's result is the following:

Obviously property $W_{p}$ implies the Banach-Saks property. Moreover, for $p>1$, the $p$-Banach-Saks property is defined as follows: A Banach space $X$ is said to have the $p$-Banach-Saks property when each bounded sequence $\left(x_{m}\right)$ admits a sub-sequence $\left(x_{n}\right)$ and a point $x$ such that $\left(x_{n}-x\right)$ is a $p$-Banach-Saks sequence, i.e., satisfies an estimate of the form

$$
\left\|\sum_{k=1}^{n} x_{k}\right\| \leq C \cdot n^{1 / p}
$$

for some constant $C>0$ and all $n \in \mathbb{N}$. It is also clear that property $W_{p}$ implies the $p^{*}$-Banach-Saks property. In [6] can be seen a proof that, conversely, the $p^{*}$-Banach-Saks property implies, for all $r>p$, the property $W_{r}$. Therefore, what this question is looking for is the extension of Núñez's result to the scale of $p$-Banach-Saks properties. 


\section{References}

[1] BOMBAL, F. Operators on spaces of vector-valued continuous functions. Extracta Math. 1, n. 3, 103-114 (1986).

[2] BOMBAL, F. On the Dunford-Pettis property. Portugaliae Math. 45, Fasc. 3, 265-272 (1988).

[3] BOMBAL, F. and CEMBRANOS, P. Characterization of some classes of operators on spaces of vector-valued continuous functions. Math. Proc. Cambridge Phil. Soc. 97, 137-146 (1985).

[4] BOMBAL, F. and RODRIGUEZ-SALINAS, B. Some classes of operators on $C(K, E)$. Extension and applications. Arch. Math. 47, 55-65 (1986).

[5] BROOKS, J.K. and LEWIS, P.W. Operators on continuous function spaces and convergence in the spaces of operators. Adv. in Math. 29, 157-177 (1978).

[6] CASTILLO, J.M.F. and SANCHEZ, F. Weackly-p-compact, p-Banach-Saks and super-reflexive Banach spaces. To appear in J. Math. Anal. and Appl.

[7] CASTILLO, J.M.F. and SANCHEZ, F. Remarks on some basic properties of Tsirelson's space. To appear in Note di Mat.

[8] CASTILLO, J.M.F. and SANCHEZ, F. Upper-l-estimates in vector sequence spaces, with some applications. Math. Proc. Cambridge Philos. Soc. 113 (1993) 329-334.

[9] CASTILLO, J.M.F. and SANCHEZ, F, Remarks on the range of a vector measure. To appear in Glasgow Math. J.

[10] CEMBRANOS, P. On Banach spaces of vector valued continuous functions. Bull. Austral. Math. Soc. 28, 175-186 (1983).

[11] DIESTEL, J. and SEIFERT, C.J. The Banach-Saks ideal, I. Operators acting on $C(\Omega)$. Commentationes Math. Tomus in honorem Ladislaw Orlicz I, 109-118 (1979).

[12] DIESTEL, J. and UHL, J.J. Jr. Vector measures. AMS Math. Surveys, 15 (1977).

[13] DOBRAKOV, I. On representation of linear operators on $C_{0}(T, X)$. Czechoslovak Math. J. 21, 13-30 (1971).

[14] HEINRICH, S. Closed operators and interpolation. J. Funct. Anal., vol. 35, n. 3, 397-411 (1980).

[15] KNAUST, H. and ODELL, E. Weakly null sequences with upper $l_{p}$-estimates. LNM 1470. Springer-Verlag.

[16] LACEY, H.E. The isometric theory of classical Banach spaces. Springer-Verlag (1974). 
[17] MADDOX, I.J. A non-absolutely summing operator. J. Austral. Math. Soc. Ser A 43 , n. 1, 70-73 (1987).

[18] NUÑEZ, C. Banach-Saks operators on spaces of continuous functions. Ann. Mat. Pura ed Appl.

[19] ROSENTHAL, H.P. Some applications of p-summing operators to Banach space theory. Studia Math. vol. 58, 21-43 (1976).

[20] SAAB, P. Weakly compact, unconditionally converging and Dunford-Pettis operators on spaces of vector-valued continuous functions. Math. Proc. Cambridge Phil. Soc. 95, 101-108 (1984).

[21) SANCHEZ, F. Sucesiones débilmente p-sumables en espacios de Banach. Pub. Dept. Mat. Univ. Extremadura vol. 28 (1991).

[22] TALAGRAND, M. La propriété de Dunford-Pettis dans $C(K, E)$ et $L_{l}(E)$. Israel J. Math. 44, 317-321 (1983).

Departamento de Matemáticas Universidad de Extremadura Avda. de Elvas, s/n 06071 Badajoz

SPAIN
Recibido: 12 de diciembre de 1991 Revisado: 6 de marzo de 1992 